\title{
STUDIES IN THE GENERAL THEORY OF RULED SURFACES*
}

\section{E. J. WILCZYNSKI $\dagger$}

The congruence $\Gamma$, which is made of all the generators of the first kind on the osculating hyperboloids of a ruled surface, has a great many interesting properties. Some of them have been considered in a previous paper. $\neq \mathrm{We}$ shall continue the consideration of this congruence and of configurations associated with it, completing in this way some of our previous investigations very essentially. We shall also study the osculating linear complex, and the pointto-plane correspondence to which it gives rise, enabling us to generalize some well-known theorems of Cremona and LiE.

For the most part we shall, in this paper, confine our attention to the general case, when the flecnode curve intersects every generator in two distinct points. The case of coincidence will be left for a future occasion, as it requires the use of a different normal form for the equations than that here adopted.

The notations are the same as in previous papers. To save space they are not again explained.

\section{$\S 1$. The derivative cubic curve.}

If $\alpha_{1}$ and $\alpha_{2}$ are arbitrary, $\alpha_{1} y_{k}+\alpha_{2} z_{k}$ will represent the coördinates of an arbitrary point on the generator $g$ of the ruled surface, where $\left(y_{k}, z_{k}\right)$ for $k=1,2,3,4$ are four simultaneous systems of solutions of our system of differential equations, whose determinant does not vanish. We shall usually write $\alpha_{1} y+\alpha_{2} z$, suppressing the index $k$, as in previous papers. Of course this is essentially a form of vector analysis, which enables us to make one equation do the work of four. The point $\alpha_{1} \rho+\alpha_{2} \sigma$ of the corresponding generator $g^{\prime}$ of $S^{\prime}$, will then be such that the line joining it to $\alpha_{1} y+\alpha_{2} z$ is a generator of the sec-

\footnotetext{
* Presented to the Society (San Francisco) December 19, 1903. Received for publication October 20, 1904.

† Of the Carnegie Institution of Washington.

$\ddagger$ On a certain congruence associated with a given ruled surface. Transactions of the A merican Mathematical Society, vol. 4 (1903), pp. 185-200, hereafter referred to as Congruence. My other papers will be quoted by the initial words of their title. See Covariants, vol. 3 (1902), p. 423, footnote.
} 
ond kind on the hyperboloid $H$ osculating $S$ along $g$. Therefore if $\beta_{1}$ and $\beta_{2}$ are arbitrary,

$$
\beta_{1}\left(\alpha_{1} y+\alpha_{2} z\right)+\beta_{2}\left(\alpha_{1} \rho+\alpha_{2} \sigma\right)
$$

will be an arbitrary point of $H$.

If we choose the tetrahedron $P_{y} P_{z} P_{\rho} P_{\sigma}$ as tetrahedron of reference for a system of homogeneous coördinates, we may take them so that the coördinates of any point represented by an expression of the form $\lambda y+\mu z+\nu \rho+\kappa \sigma$ will be $x_{1}=\lambda, x_{2}=\mu, x_{3}=\nu, x_{4}=\kappa$. We have then

$$
x_{1}=\beta_{1} \alpha_{1}, \quad x_{2}=\beta_{1} \alpha_{2}, \quad x_{3}=\beta_{2} \alpha_{1}, \quad x_{4}=\beta_{2} \alpha_{2}
$$

as the coördinates of an arbitrary point on $H$, and therefore

$$
x_{1} x_{4}-x_{2} x_{3}=0
$$

as the equation of $H$ in this system of coördinates.

Let us consider now the hyperboloid $H^{\prime}$, which osculates $S^{\prime}$ along $g^{\prime}$. The coördinates of $P_{\rho}$ and $P_{\sigma}$ were obtained from the system of differential equations defining $S$ by forming

$$
\rho=2 y^{\prime}+p_{11} y+p_{12} z, \quad \sigma=2 z^{\prime}+p_{21} y+p_{22} z .
$$

We shall obtain the coördinates of two points on a generator $g^{\prime \prime}$ of the derivative of $S^{\prime}$ with respect to $x$, by applying the same process to the equations [Congruence, (15)] which define $S^{\prime}$. The ruled surface $S^{\prime \prime}$ thus obtained shall be naturally called the second derivative of $S$ with respect to $x$. Its generator $g^{\prime \prime}$ is then a generator of the hyperboloid $H^{\prime}$ which osculates $S^{\prime}$ along $g^{\prime}$. The following quantities

$$
\begin{aligned}
& 2 \rho^{\prime}+P_{11} \rho+P_{12} \sigma=u_{11} y+u_{12} z+\lambda_{11} \rho+\lambda_{12} \sigma, \\
& 2 \sigma^{\prime}+P_{21} \rho+P_{22} \sigma=u_{21} y+u_{22} z+\lambda_{21} \rho+\lambda_{22} \sigma
\end{aligned}
$$

are the coördinates of two points on $g^{\prime \prime}$.

These equations show that $g^{\prime \prime}$ intersects $g$, if and only if $\lambda_{11} \lambda_{22}-\lambda_{12} \lambda_{21}=0$, i. e., if $K=v_{11} v_{22}-v_{12} v_{21}=0$, provided we assume that $S^{\prime}$ is not developable. By changing the independent variable one can always change $K$ into $\bar{K}$ such that $\bar{K}=0$. The equation for $\eta$, [Congruence, equ. (4)], which must be satisfied so as to make $\bar{K}=0$, is of the second order. Therefore there exist $\infty^{2}$ nondevelopable ruled surfaces in the congruence $\Gamma$, each of which, when considered as the first derivative of $S$, gives rise to a second derivative whose generators intersect the corresponding generators of $S$.

Let us consider any point on $g^{\prime}$, whose coördinates are $\epsilon_{1} \rho+\varepsilon_{2} \sigma$. The corresponding point on $g^{\prime \prime}$ will be given by

$$
\left(\epsilon_{1} u_{11}+\epsilon_{2} u_{21}\right) y+\left(\epsilon_{1} u_{12}+\epsilon_{2} u_{22}\right) z+\left(\epsilon_{1} \lambda_{11}+\epsilon_{2} \lambda_{21}\right) \rho+\left(\epsilon_{1} \lambda_{12}+\epsilon_{2} \lambda_{22}\right) \sigma .
$$


Therefore, the expression

$$
\begin{aligned}
\delta_{1}\left(\epsilon_{1} u_{11}+\epsilon_{2} u_{21}\right) y & +\delta_{1}\left(\epsilon_{1} u_{12}+\epsilon_{2} u_{22}\right) z \\
& +\left[\delta_{1}\left(\epsilon_{1} \lambda_{11}+\epsilon_{2} \lambda_{21}\right)+\delta_{2} \epsilon_{1}\right] \rho+\left[\delta_{1}\left(\epsilon_{1} \lambda_{12}+\epsilon_{2} \lambda_{22}\right)+\delta_{2} \epsilon_{2}\right] \sigma
\end{aligned}
$$

will, for arbitrary values of $\epsilon_{1}, \epsilon_{2}, \delta_{1}, \delta_{2}$, represent an arbitrary point of $H^{\prime}$. If we introduce again our special system of coördinates we have

$$
\begin{array}{ll}
x_{1}=u_{11} \delta_{1} \epsilon_{1}+u_{21} \delta_{1} \epsilon_{2}, & x_{3}=\lambda_{11} \delta_{1} \epsilon_{1}+\lambda_{21} \delta_{1} \epsilon_{2}+\delta_{2} \epsilon_{1}, \\
x_{2}=u_{12} \delta_{1} \epsilon_{1}+u_{22} \delta_{1} \epsilon_{2}, & x_{4}=\lambda_{21} \delta_{1} \epsilon_{1}+\lambda_{22} \delta_{1} \epsilon_{2}+\delta_{2} \epsilon_{2},
\end{array}
$$

as the coördinates of an arbitrary point of $H^{\prime}$. If we eliminate $\delta_{1}, \delta_{2}, \epsilon_{1}, \epsilon_{2}$, we find the equation of $H^{\prime}$ :

$$
\begin{aligned}
& \left(u_{22} x_{1}-u_{21} x_{2}\right)\left[J x_{4}-\left(\lambda_{12} u_{22}-\lambda_{22} u_{12}\right) x_{1}+\left(\lambda_{12} u_{21}-\lambda_{22} u_{11}\right) x_{2}\right] \\
& \quad+\left(u_{12} x_{1}-u_{11} x_{2}\right)\left[J x_{3}-\left(\lambda_{11} u_{22}-\lambda_{21} u_{12}\right) x_{1}+\left(\lambda_{11} u_{21}-\lambda_{21} u_{11}\right) x_{2}\right]=0 .
\end{aligned}
$$

We shall mostly assume that $P_{y}$ and $P_{z}$ are the flecnodes of $g$, so that $u_{12}=u_{21}=0$, and (3) simplifies into

$$
(3 a)-\lambda_{12} u_{22}^{2} x_{1}^{2}+\lambda_{21} u_{11}^{2} x_{2}^{2}+u_{11} u_{22}\left(\lambda_{11}-\lambda_{22}\right) x_{1} x_{2}+u_{11} u_{22}\left(u_{22} x_{1} x_{4}-u_{11} x_{2} x_{3}\right)=0 \text {. }
$$

It is easy to see from $(3 a)$ that $H^{\prime}$ cannot coincide with $H$, unless $S$ is a quadric.

The hyperboloids $H$ and $H^{\prime}$ have the straight line $g^{\prime}$ in common. The rest of their intersection is therefore, in general, a space cubic. We shall call it the derivative cubic, and discuss some of its properties. It is interesting to notice that we obtain in this way associated with every ruled surface, a surface containing a single infinity of twisted cubics. This surface we shall also consider.

Let us again assume that $P_{y}$ and $P_{z}$ are the flecnodes of $g$. Then, it follows from $(1)$ and $(3 a)$ that we may take

$$
\begin{aligned}
& x_{1}=t x_{2}, \quad x_{2}=J\left(u_{11}-u_{22}\right) t, \quad x_{3}=t x_{4}, \\
& x_{4}=-\lambda_{12} u_{22}^{2} t^{2}+u_{11} u_{22}\left(\lambda_{11}-\lambda_{22}\right) t+\lambda_{21} u_{11}^{2}
\end{aligned}
$$

as the parametric equations of the cubic. From these, the following corollaries follow at once. If $\lambda_{11}-\lambda_{22}=0$, the derivative cubic, and therefore the hyperboloid $H^{\prime}$, intersects $g$ in two points, which are harmonic conjugates with respect to the flecnodes. If

$$
\left(\lambda_{11}-\lambda_{22}\right)^{2}+4 \lambda_{12} \lambda_{21}=0
$$

the cubic is tangent to $g$. The congruence $\Gamma$ contains $\infty^{2}$ surfaces $S^{\prime}$ corresponding to each of these properties of the derivative cubic. For, the corresponding equations for $\eta$ are again of the second order.

The equation of a plane which is tangent to $H^{\prime}$ at a point $\left(x_{1}^{\prime}, x_{2}^{\prime}, x_{3}^{\prime}, x_{4}^{\prime}\right)$, $P_{y}$ and $P_{z}$ being flecnodes, is 


$$
\begin{aligned}
& {\left[-2 \lambda_{12} u_{22}^{2} x_{1}^{\prime}+u_{11} u_{22}\left(\lambda_{11}-\lambda_{22}\right) x_{2}^{\prime}+u_{11} u_{22}^{2} x_{4}^{\prime}\right] x_{1}} \\
& +\left[u_{11} u_{22}\left(\lambda_{11}-\lambda_{22}\right) x_{1}^{\prime}+2 \lambda_{21} u_{11}^{2} x_{2}^{\prime}-u_{11}^{2} u_{22} x_{3}^{\prime}\right] x_{2} \\
& -u_{11}^{2} u_{22} x_{2}^{\prime} x_{3}+u_{11} u_{22}^{2} x_{1}^{\prime} x_{4}=0 .
\end{aligned}
$$

Consider the two points of $g^{\prime \prime}$ which correspond to $P_{y}$ and $P_{z}$, viz.,

$$
P_{1}^{\prime \prime}=\left(u_{11}, 0, \lambda_{11}, \lambda_{12}\right), \quad P_{2}^{\prime \prime}=\left(0, u_{22}, \lambda_{21}, \lambda_{22}\right) \text {. }
$$

The equations of the two planes tangent to $S^{\prime \prime}$ or $H^{\prime}$ at these points respectively, are

$$
-u_{22} \lambda_{12} x_{1}-u_{11} \lambda_{22} x_{2}+u_{11} u_{22} x_{4}=0, \quad u_{22} \lambda_{11} x_{1}+u_{11} \lambda_{21} x_{2}-u_{11} u_{22} x_{3}=0 .
$$

They intersect $g$, i. e., the line $x_{3}=x_{4}=0$, in two points

$$
P_{1}=\left(u_{11} \lambda_{22},-u_{22} \lambda_{12}, 0,0\right), \quad P_{2}=\left(u_{11} \lambda_{21},-u_{22} \lambda_{11}, 0,0\right),
$$

which coincide, if and only if $\lambda_{11} \lambda_{22}-\lambda_{12} \lambda_{21}=0$, i. e., if and only if $g^{\prime \prime}$ intersects $g$, as is moreover geometrically evident. $P_{1}$ and $P_{2}$ are harmonic conjugates with respect to the flecnodes if $\lambda_{11} \lambda_{22}+\lambda_{12} \lambda_{21}=0$, i. e., under this condition the planes, tangent to the second derivative at the points which correspond to the flecnodes of $g$, divide these flecnodes harmonically.

The line $P_{z} P_{\rho}$ has, besides $P_{\rho}$, another point in common with $H^{\prime}$. Its coördinates are found to be $\left(0, u_{22}, \lambda_{21}, 0\right)$. Similarly $P_{y} P_{\sigma}$ intersects $H^{\prime}$ in a second point $\left(u_{11}, 0,0, \lambda_{12}\right)$. Join these two points. The coördinates of any point on this line joining them will be $\left(\mu u_{11}, \lambda u_{22}, \lambda \lambda_{21}, \mu \lambda_{12}\right)$, where $\lambda: \mu$ determines the position of the particular point. It is easily seen, by substituting in $(3 a)$, that this line is entirely on $H^{\prime}$ if $\lambda_{11}-\lambda_{22}=0$, and in no other case, provided that $S^{\prime}$ is not developable. We have therefore the following result. Corresponding to every point of $S$ we have a point of $S^{\prime}$. If each of the two flecnodes on a generator $g$ of $S$ be joined to the point of $S^{\prime}$ which corresponds to the other, the two straight lines thus obtained intersect the hyperboloid $H^{\prime}$ osculating $S^{\prime}$ along $g^{\prime}$ in two new points. The line joining these latter points lies entirely on $H^{\prime}$, if $H^{\prime}$ intersects $g$ in two points which are harmonic conjugates with respect to the flecnodes. The converse of the theorem is also true.

Let us introduce the following abbreviations:

$$
\begin{aligned}
& A=2 u_{11} u_{22}\left(u_{11}-u_{22}\right), \quad \psi=B t_{1}^{2}+2 C t_{1} t_{2}+D t_{2}^{2}, \\
& B=v_{12} u_{22}, \quad C=\frac{1}{2}\left(u_{11} v_{22}-u_{22} v_{11}\right), \quad D=-v_{21} u_{11},
\end{aligned}
$$

and let us write the parameter $t$ of the cubic curve in homogeneous form. Then we may write instead of (4),

$$
\begin{array}{ll}
x_{1}=A t_{1}^{2} t_{2}, & x_{3}=\left(B t_{1}^{2}+2 C t_{1} t_{2}+D t_{2}^{2}\right) t_{1}=\psi t_{1}, \\
x_{2}=A t_{1} t_{2}^{2}, & x_{4}=\left(B t_{1}^{2}+2 C t_{1} t_{2}+D t_{2}^{2}\right) t_{2}=\psi t_{2} .
\end{array}
$$


If the cubic degenerates, each irreducible part will be a plane curve (a conic or a straight line). If therefore the cubic degenerates it must be possible to satisfy the equation

$$
a_{1} x_{1}+a_{2} x_{2}+a_{3} x_{3}+a_{4} x_{4}=0,
$$

for all values of $t_{1}: t_{2}$, the coefficients being independent of $t_{1}: t_{2}$. If we substitute in this equation the values (7), and equate to zero the coefficients of $t_{1}^{3}, t_{1}^{2} t_{2}$, etc., we find

$$
a_{3} B=0, a_{4} D=0, a_{1} A+2 a_{3} C+a_{4} B=0, a_{2} A+a_{3} D+2 a_{4} C=0 .
$$

Let us assume first that neither $B$ nor $D$ vanishes. Then $a_{3}=a_{4}=0$, and $A=0$, for if $A$ were not zero, we would have also $a_{1}=a_{2}=0$, i. e., there would be no plane containing the (supposedly) degenerate cubic. But from $A=0$ follows either $u_{11}-u_{22}=0$, which would make $S$ a quadric, or else $u_{11}$ or $u_{22}$ would vanish, which however contradicts the assumption that $B$ and $D$ shall not be zero.

Let us now assume $B \neq 0, D=0$. Then $a_{3}=0$, and either $u_{11}$ or $v_{21}$ must vanish, i. e., either $S^{\prime}$ is developable or $S$ has a straight line directrix. Similarly if $B=0, D \neq 0$. Finally if $B=0, D=0$, either $S$ has two straight line directrices, or else it has one while $S^{\prime}$ is developable. We have therefore the following theorem. If the surface has one or more straight line directrices the derivative cubic always degenerates. In all other cases, the only way to obtain a degenerate derivative cubic consists in taking as derivative ruled surface of $S$, one of the developables of the congruence $\Gamma$.

Another question at once suggests itself. To every value of $x$, i. e., to every generator of $S$ there belongs a derivative cubic. In general, the cubics belonging to values of $x$, differing from each other by an infinitesimal $\delta x$, will not intersect. Their shortest distance will be an infinitesimal of the same order as $\delta x$. It may happen however that, for an appropriately chosen variable, this distance becomes infinitesimal of a higher order, or as we may say briefly, that consecutive cubics intersect. We ask now : is it possible to choose the independent variable in such a way that every pair of consecutive derivative cubics may intersect?

By putting $y=y_{k}, z=z_{k}(k=1,2,3,4)$ in

$$
\phi=A t_{1} t_{2}\left(t_{1} y+t_{2} z\right)+\psi\left(t_{1} \rho+t_{2} \sigma\right),
$$

we obtain the coördinates of any point $P_{\phi}$ on the cubic. As $x$ changes we go from one cubic to another; as $t_{1}: t_{2}$ changes we go from one point on a certain cubic to another point of the same curve. Equation (9) gives therefore, if both $x$ and $t_{1}: t_{2}$ be taken as variables, the locus of all such points $P_{\phi}, i$. e., the surface generated by all of the derivative cubics of $S$. If $t_{1}: t_{2}$ be chosen as a function of $x$, a curve is picked out upon this surface. Let us differentiate $\phi$ 
totally, i. e., assuming that $t_{1}$ and $t_{2}$ are functions of $x$, and consider the quantity

$$
\phi+\frac{d \phi}{d x} \delta x,
$$

where $\delta x$ is an infinitesimal. This will clearly represent the coördinates of a point on the adjacent derivative cubic determined by the parameters $x+\delta x$ and $t_{k}+d t_{k} / d x \delta x$. If the original cubic and this second one, infinitesimally close to it, intersect, it must be possible to choose $t_{k}$ as functions of $x$ in such a way, that the corresponding points of the two curves coincide up to infinitesimals of higher than the first order. Therefore $d \phi / d x$ must differ from a multiple of $\phi$ only by an infinitesimal quantity. Proceeding to the limit we must therefore have

We find by differentiation

$$
\frac{d \phi}{d x}=\omega \phi
$$

$$
\begin{aligned}
\frac{d \phi}{d x}=[( & \left.\left.t_{1} t_{2}^{\prime}+t_{1}^{\prime} t_{2}\right) A+A^{\prime} t_{1} t_{2}\right]\left(t_{1} y+t_{2} z\right) \\
& +y\left[A t_{1} t_{2}\left(t_{1}^{\prime}-\frac{1}{2} p_{11} t_{1}-\frac{1}{2} p_{21} t_{2}\right)+\frac{1}{2} \psi u_{11} t_{1}\right] \\
& +z\left[A t_{1} t_{2}\left(t_{2}^{\prime}-\frac{1}{2} p_{12} t_{1}-\frac{1}{2} p_{22} t_{2}\right)+\frac{1}{2} \psi u_{22} t_{2}\right] \\
& +\rho\left[\frac{1}{2} A t_{1}^{2} t_{2}+\psi\left(t_{1}^{\prime}-\frac{1}{2} p_{11} t_{1}-\frac{1}{2} p_{21} t_{2}\right)+\psi^{\prime} t_{1}\right] \\
& +\sigma\left[\frac{1}{2} A t_{1} t_{2}^{2}+\psi\left(t_{2}^{\prime}-\frac{1}{2} p_{12} t_{1}-\frac{1}{2} p_{22} t_{2}\right)+\psi^{\prime} t_{2}\right],
\end{aligned}
$$

denoting as usual differentiation by strokes.

If we substitute in (10) we find the following four equations:

(a) $\left[\left(t_{1} t_{2}^{\prime}+t_{1}^{\prime} t_{2}\right) A+A^{\prime} t_{1} t_{2}\right] t_{1}+A t_{1} t_{2}\left(t_{1}^{\prime}-\frac{1}{2} p_{11} t_{1}-\frac{1}{2} p_{21} t_{2}\right)$

$$
+\frac{1}{2} \psi u_{11} t_{1}=\omega A t_{1}^{2} t_{2}
$$

(b) $\left[\left(t_{1} t_{2}^{\prime}+t_{1}^{\prime} t_{2}\right) A+A^{\prime} t_{1} t_{2}\right] t_{2}+A t_{1} t_{2}\left(t_{2}^{\prime}-\frac{1}{2} p_{12} t_{1}-\frac{1}{2} p_{22} t_{2}\right)$

$$
+\frac{1}{2} \psi u_{22} t_{2}=\omega A t_{1} t_{2}^{2}
$$

(c) $\frac{1}{2} A t_{1}^{2} t_{2}+\psi\left(t_{1}^{\prime}-\frac{1}{2} p_{11} t_{1}-\frac{1}{2} p_{21} t_{2}\right)+\psi^{\prime} t_{1}=\omega \psi t_{1}$,

(d) $\frac{1}{2} A t_{1} t_{2}^{2}+\psi\left(t_{2}^{\prime}-\frac{1}{2} p_{12} t_{1}-\frac{1}{2} p_{22} t_{2}\right)+\psi^{\prime} t_{2}=\omega \psi t_{2}$.

If we multiply both members of $(a)$ by $t_{2}$, of $(b)$ by $-t_{1}$, and add, and if we treat $(c)$ and $(d)$ in the same way, we find

$$
\begin{gathered}
\text { (e) } A t_{1} t_{2}^{2}\left(t_{1}^{\prime}-\frac{1}{2} p_{11} t_{1}-\frac{1}{2} p_{21} t_{2}\right)-A t_{1}^{2} t_{2}\left(t_{2}^{\prime}-\frac{1}{2} p_{12} t_{1}-\frac{1}{2} p_{22} t_{2}\right) \\
+\frac{1}{2} \psi t_{1} t_{2}\left(u_{11}-u_{22}\right)=0, \\
\text { (f) } \psi t_{2}\left(t_{1}^{\prime}-\frac{1}{2} p_{11} t_{1}-\frac{1}{2} p_{21} t_{2}\right)-\psi t_{1}\left(t_{2}^{\prime}-\frac{1}{2} p_{12} t_{1}-\frac{1}{2} p_{22} t_{2}\right)=0 .
\end{gathered}
$$


Let us assume first $\psi \neq 0$. Divide both members of $(f)$ by $\psi$, and compare with $(e)$. We find

$$
\psi t_{1} t_{2}\left(u_{11}-u_{22}\right)=0,
$$

i. e., either $S$ is a quadric, or else either $t_{1}$ or $t_{2}$ must vanish identically. Assume $t_{1}=0$. Then $(e)$ is satisfied. From $(f)$ we would find $p_{21}=0$, for $t_{2}$ cannot be zero, since the ratio $t_{1}: t_{2}$ is the parameter which determines a point on the cubic. But from $u_{21}=p_{21}=0$ follows further $v_{21}=0$, i. e., $D=0$, so that in this case $\psi=B t_{1}^{2}+2 C t_{1} t_{2}=0$ contrary to our assumption. It is therefore impossible to satisfy (11) except by putting $\psi=0$. According to (c) and $(d)$ this gives either $t_{1}=0$, or $t_{2}=0$, or $A=0$. Assume $A \neq 0$ and say $t_{1}=0$. All equations (11) are now satisfied. But from $t_{1}=0, \psi=0$ follows $D=0$, i. e., either $u_{11}$ or $v_{21}$ must vanish, i. e., either $S$ has a straight line directrix or else $S^{\prime}$ is developable. If however $A=0$, either $S$ is a quadric or else $S^{\prime}$ is developable.

In connection with our last theorem, we may therefore say: It is impossible to choose the surface $S^{\prime}$ of the congruence $\Gamma$ in such a way that consecutive derivative cubics may intersect, except in the trivial cases when the cubics are degenerate.

If in (9) we put $\psi=0$, we obtain the locus of the intersections of the cubic with the generator of $S$ to which it belongs, i. e., a certain curve cutting every generator twice. This may be an asymptotic curve. It is, in fact, if the further conditions

$$
2 t_{1}^{\prime}-p_{11} t_{1}-p_{21} t_{2}=\omega t_{1}, \quad 2 t_{2}^{\prime}-p_{12} t_{1}-p_{22} t_{2}=\omega t_{2}
$$

are satisfied, where $\omega$ is arbitrary. For, as $(10 a)$ shows, the line joining $P_{\phi}$ to $P_{\phi+\phi^{\prime} \delta x}$, i. e., the tangent to this curve, is then a generator of the second kind on the hyperboloid osculating $S$ along $g$. In other words it is a tangent to an asymptotic curve of $S$.

In general, of course the conditions (12) and $\psi=0$ can not both be satisfied at once. The question is: when are these conditions consistent? We find from (12)

$$
t_{1}^{\prime}=\frac{1}{2}\left[\left(p_{11}+\omega\right) t_{1}+p_{21} t_{2}\right], \quad t_{2}^{\prime}=\frac{1}{2}\left[p_{12} t_{1}+\left(p_{22}+\omega\right) t_{2}\right] .
$$

Let us substitute these values of $t_{1}^{\prime}$ and $t_{2}^{\prime}$ in the equation $d \psi / d x=0$. We shall find

$$
\begin{aligned}
{\left[B^{\prime}+B\left(p_{11}+\omega\right)+\right.} & \left.C p_{12}\right] t_{1}^{2}+\left[D^{\prime}+D\left(p_{22}+\omega\right)+C p_{21}\right] t_{2}^{2} \\
& +\left[2 C^{\prime}+B p_{12}+D p_{21}+C\left(p_{11}+p_{22}+2 \omega\right)\right] t_{1} t_{2}=0,
\end{aligned}
$$

which compared with $\psi=0$ gives,

$$
B^{\prime}+B\left(p_{11}+\omega\right)+C p_{12}=\tau B
$$




$$
\begin{gathered}
D^{\prime}+D\left(p_{22}+\omega\right)+C p_{21}=\tau D, \\
2 C^{\prime}+B p_{12}+D p_{21}+C\left(p_{11}+p_{22}+2 \omega\right)=2 \tau C,
\end{gathered}
$$

where $\tau$ is a proportionality factor. Eliminating $\tau$, we find

(a) $2\left(B^{\prime} C-B C^{\prime}\right)+B C\left(p_{11}-p_{22}\right)+\left(2 C^{2}-B D\right) p_{12}-B^{2} p_{21}=0$,

(b) $2\left(D^{\prime} C-D C^{\prime}\right)+D C\left(p_{22}-p_{11}\right)+\left(2 C^{2}-B D\right) p_{21}-D^{2} p_{12}=0$,

(c) $B D^{\prime}-B^{\prime} D+B D\left(p_{11}-p_{22}\right)+C\left(D p_{12}-B p_{21}\right)=0$.

We may always assume that our system of differential equations has been so written that $p_{11}-p_{22}=0$. Equations (13) may then be regarded as three homogeneous linear equations for $1, p_{12}, p_{21}$. Their determinant must therefore be zero. This gives rise to three alternatives : either $C^{2}-B D$ or $C$ or $B D^{\prime}-B^{\prime} D$ must vanish.

Consider first the case $C^{2}-B D=0$. Equations (13) become

$$
\begin{gathered}
2\left(B^{\prime} C-B C^{\prime}\right)-B^{2} p_{21}=0, \quad 2\left(D^{\prime} C-D C^{\prime}\right)-D^{2} p_{12}=0, \\
B D^{\prime}-B^{\prime} D+C D p_{12}-C B p_{21}=0 .
\end{gathered}
$$

If we multiply both members of the first two equations by $C D$ and $-C B$ respectively, add and make use of $C^{2}=B D$, we find

$$
2 C^{2}\left(D B^{\prime}-D^{\prime} B\right)+C^{2}\left(C D p_{12}-C B p_{21}\right)=0,
$$

whence, if $C \neq 0$,

$$
-2\left(B D^{\prime}-B^{\prime} D\right)+C D p_{12}-C B p_{21}=0 .
$$

But if we consider the last equation of (14), this gives $B D^{\prime}-B^{\prime} D=0$, $D p_{12}-B p_{21}=0$, which last equation may be written

$$
p_{12} p_{21}\left(u_{11}-u_{22}\right)^{2}=0,
$$

i. e., the surface $S$ has at least one straight line directrix.

In the second place let $C=0$. Then (13) becomes

$$
B\left(D p_{12}+B p_{21}\right)=0, \quad D\left(D p_{12}+B p_{21}\right)=0, \quad B D^{\prime}-B^{\prime} D=0 .
$$

According to the first two equations $S$ must either have at least two straight line directrices, or else $u_{11}+u_{22}$ must be zero. The third condition gives, on substituting the values of $B$ and $D$,

$$
\frac{u_{22}^{\prime}}{u_{22}}-\frac{u_{11}^{\prime}}{u_{11}}+\frac{v_{12}^{\prime}}{v_{12}}-\frac{v_{21}^{\prime}}{v_{21}}=0
$$

if we assume that $S$ has no straight line directrix, so that $v_{12}$ and $v_{21}$ are not 
zero. By integration we find

$$
\frac{v_{12}}{v_{21}} \frac{u_{22}}{u_{11}}=\text { const., }
$$

whence, since $u_{11}+u_{22}=0$ in this case,

$$
\frac{p_{12}}{p_{21}}=\text { const. }
$$

But if we substitute into the condition $\Delta=0$ for a surface belonging to a linear complex the assumptions $u_{12}=u_{21}=p_{11}-p_{22}=0$, which we have made, we find either $u_{11}-u_{22}=0$, i. e., $S$ is a quadric, or $p_{12} p_{21}^{\prime}-p_{12}^{\prime} p_{21}=0$, i. e., $p_{12} / p_{21}=$ const. Therefore we see that if $C=0, S$ either has straight line directrices, or at least belongs to a linear complex. Moreover in that case the independent variable must be so chosen as to make $u_{11}+u_{22}$ vanish.

If finally $B D^{\prime}-B^{\prime} D=0$, we find from (13) either $C=0$, which leads us back to the case just considered, or else $D p_{12}-B p_{21}$ must vanish, which gives again a surface $S$ with at least one straight line directrix.

In all of these cases the surface $S$ belongs to a linear complex. In the special cases the asymptotic lines thus obtained are merely two of the straight line directrices, if there are two, or else one straight line directrix and another curved asymptotic line obtained by putting $u_{11}+u_{22}=0$. If we leave aside the trivial cases, we can say that if a ruled surface belongs to a linear complex, and if the independent variable is so chosen that $u_{11}+u_{22}=0$, the surface of derivative cubics determines an asymptotic curve upon it which intersects every generator twice.

We have seen (Congruence, p. 197), that there exists in the congruence $\Gamma$ a single infinity of ruled surfaces $S^{\prime}$ for which $u_{11}+u_{22}=0$. They are those ruled surfaces of $\Gamma$ whose intersections with the flecnode surface of $S$ are asymptotic lines upon them. But we can also characterize them by saying that such a surface is made up of the lines of $\Gamma$ intersecting any asymptotic curve on the flecnode surface of $S$. In fact, if we assume $u_{12}=u_{21}=0, p_{11}=p_{22}=0$, we may write the equations of the sheet $F^{\prime}$ of the flecnode surface as follows: *

$$
\begin{gathered}
y^{\prime \prime}-2{ }_{p_{12}}^{q_{12}} y^{\prime}-\rho^{\prime}-q_{11} y+\frac{q_{12}}{p_{12}} \rho=0, \\
\rho^{\prime \prime}+\left[2\left(q_{11}+q_{22}\right)-p_{12} p_{21}\right] y^{\prime}-2 \frac{q_{12}}{p_{12}} \rho^{\prime}+\left[2 q_{11}^{\prime}-p_{12} q_{21}-4 \frac{q_{12}}{p_{12}} q_{11}\right] y-q_{22} \rho=0 .
\end{gathered}
$$

If $u_{11}+u_{22}=0$, the coefficient of $y^{\prime}$ in the second equation vanishes, which proves that the curve $C_{\rho}$ is an asymptotic curve on $F^{\prime}$. The asymptotic curves

* The system of equations for the flecnode surface, in Congruence, p. 190, equ. (12), does not agree exactly with (15) because there the further assumption $u_{11}=0$ was made. This should have been stated there. 
on $F^{\prime}$ and $F^{\prime \prime}$ must therefore correspond to each other. The congruence $\Gamma$ is therefore a so-called $W$-congruence. *

We may now state our theorem as follows: In order that the surface of derivative cubics may intersect the ruled surface $S$ in an asymptotic curve, $S$ must belong to a linear complex. Moreover, its derivative ruled surface must intersect the flecnode surface of $S$ along an asymptotic curve.

The asymptotic curve on $S$, thus determined, is unique. For the ratio $B: D$ which determines it cannot be changed by any transformation of the variables subject to the conditions $p_{11}-p_{22}=u_{12}=u_{21}=u_{11}+u_{22}=0$. We may then take any asymptotic curve of the flecnode surface and consider the ruled surface of $\Gamma$ made up of the lines which intersect it. We obtain thus as a consequence a single infinity of surfaces made up of derivative cubics. All of of these intersect $S$ along the same asymptotic curve.

But we notice further that $C=0$. Therefore, this asymptotic curve intersects every generator in two points which are harmonic conjugates with respect to the flecnodes. We shall meet this special asymptotic curve again in a later paragraph.

Another question suggests itself. Is it possible to choose the independent variable of our system in such a way that the derivative cubics shall be asymptotic lines on the surface generated by them?

In order to answer this question, we must first find the coördinates of the osculating plane of the cubic at any one of its points. For the moment we prefer to take the equation of the cubic referred to a non-homogeneous parameter $t$, i. e., in the form

$x_{1}=A t^{2}, \quad x_{2}=A t, \quad x_{3}=B t^{3}+2 C t^{2}+D t, \quad x_{t}=B t^{2}+2 C t+D$.

Its intersections with the plane $\sum u_{i} x_{i}=0$, whose coördinates are $\left(u_{1}, \cdots u_{1}\right)$, will be given by solving the cubic equation

$$
B u_{3} t^{3}+\left(A u_{1}+2 C u_{3}+B u_{4}\right) t^{2}+\left(A u_{2}+2 C u_{4}+D u_{3}\right) t+D u_{4}=0 .
$$

The three roots of this cubic must coincide if the plane is an osculating plane of the cubic curve. They must therefore also satisfy the equations obtained from the above by twofold differentiation with respect to $t$. This gives the following conditions :

$$
\begin{aligned}
3 B u_{3} t+A u_{1}+2 C u_{3}+B u_{4} & =0 \\
\left(A u_{1}+2 C u_{3}+B u_{4}\right) t+A u_{2}+2 C u_{4}+D u_{3} & =0 \\
\left(A u_{2}+2 C u_{4}+D u_{3}\right) t+3 D u_{4} & =0
\end{aligned}
$$

Of course, only the ratios of $u_{1} \cdots u_{4}$ are of interest, so that we may multiply $u_{1} \cdots u_{4}$ by a common factor if we please. We find

* Cf. Bianchi-Lukat, Vorlesungen über Differentialgeometrie, p. 315. 


$$
\begin{array}{ll}
u_{1}=B^{2} t^{3}-3 B D t-2 C D, & u_{3}=A D, \\
u_{2}=2 B C t^{3}+3 B D t^{2}-D^{2}, & u_{4}=-A B t^{3},
\end{array}
$$

as the coördinates of the plane osculating the derivative cubic belonging to the generator $g$ at the point whose parameter is $t$, or in homogeneous form,

$$
\begin{array}{ll}
u_{1}=B^{2} t_{1}^{3}-3 B D t_{1} t_{2}^{2}-2 C D t_{2}^{3}, & u_{3}=A D t_{2}^{3}, \\
u_{2}=2 B C t_{1}^{3}+3 B D t_{1}^{2} t_{2}-D^{2} t_{2}^{3}, & u_{4}=-A B t_{1}^{3} .
\end{array}
$$

We have already made use of the expression

$$
\phi=A t_{1} t_{2}\left(t_{1} y+t_{2} z\right)+\psi\left(t_{1} \rho+t_{2} \sigma\right)
$$

as giving the coördinates of a point $\left(t_{1}: t_{2}\right)$ of the cubic curve which belongs to the argument $x$, or as giving the coördinates of a point $\left(x, t_{1}: t_{2}\right)$ of the surface formed by the aggregate of all of these curves. The plane which is tangent to this surface at the point $\left(x, t_{1}: t_{2}\right)$ must contain also the point $t_{1}: t_{2}$ of the adjacent cubic, i. e., the point whose coördinates are given by

$$
\phi+\frac{\partial \phi}{\partial x} \delta x
$$

where in forming $\partial \phi / \partial x, x, t_{1}$ and $t_{z}$ are regarded as independent variables. The tangent plane must therefore contain the point whose coördinates are $\partial \phi / \partial x$. We have

$$
\begin{aligned}
\frac{\partial \phi}{\partial x}=y[ & \left.A^{\prime} t_{1}^{2} t_{2}-\frac{1}{2} A t_{1} t_{2}\left(p_{11} t_{1}+p_{21} t_{2}\right)+\frac{1}{2} \psi u_{11} t_{1}\right] \\
& +z\left[A^{\prime} t_{1} t_{2}^{2}-\frac{1}{2} A t_{1} t_{2}\left(p_{12} t_{1}+p_{22} t_{2}\right)+\frac{1}{2} \psi u_{22} t_{2}\right] \\
& +\rho\left[\frac{1}{2} A t_{1}^{2} t_{2}-\frac{1}{2} \psi\left(p_{11} t_{1}+p_{21} t_{2}\right)+\frac{\partial \psi}{\partial x} t_{1}\right] \\
& +\sigma\left[\frac{1}{2} A t_{1} t_{2}^{2}-\frac{1}{2} \psi\left(p_{12} t_{1}+p_{22} t_{2}\right)+\frac{\partial \psi}{\partial x} t_{2}\right] .
\end{aligned}
$$

The point, whose coördinates $\left(\xi_{1}, \ldots, \xi_{4}\right)$ are the coefficients of $y, z, \rho, \sigma$ in this expression, must be in the tangent plane of the point $\left(x, t_{1}: t_{2}\right)$. If therefore, the cubic curve is an asymptotic line upon the surface, its osculating plane must contain the point $\left(\xi_{1}, \ldots, \xi_{4}\right)$, i. e., we must have

$$
u_{1} \xi_{1}+u_{2} \xi_{2}+u_{3} \xi_{3}+u_{4} \xi_{4}=0 \text {. }
$$

We find in the first place

$$
\begin{aligned}
& \xi_{1}=u_{11} B t_{1}^{3}+\left(2 A^{\prime}+2 u_{11} C-A p_{11}\right) t_{1}^{2} t_{2}+\left(u_{11} D-A p_{21}\right) t_{1} t_{2}^{2}, \\
& \xi_{2}=u_{22} D t_{2}^{3}+\left(u_{22} B-A p_{12}\right) t_{1}^{2} t_{2}+\left(2 A^{\prime}+2 u_{22} C-A p_{22}\right) t_{1} t_{2}^{2},
\end{aligned}
$$


(19)

$$
\begin{aligned}
\xi_{3}=\left(2 B^{\prime}-B p_{11}\right) t_{1}^{3}+\left(A-B p_{21}\right. & \left.+4 C^{\prime}-2 C p_{11}\right) t_{1}^{2} t_{2} \\
& +\left(2 D^{\prime}-D p_{11}-2 C p_{21}\right) t_{1} t_{2}^{2}-D p_{21} t_{2}^{3}, \\
\xi_{4}=-B p_{12} t_{1}^{3}+ & \left(2 B^{\prime}-B p_{22}-2 C p_{12}\right) t_{1}^{2} t_{2} \\
+\left(A-D p_{12}\right. & \left.+4 C^{\prime}-2 C p_{22}\right) t_{1} t_{2}^{2}+\left(2 D^{\prime}-D p_{22}\right) t_{2}^{3} .
\end{aligned}
$$

If these values, and the values (17) for $u_{1}, \ldots, u_{4}$ be substituted in (18), and the coefficients of $t_{1}^{6}, t_{1}^{5} t_{2}$, etc., be successively equated to zero, the following seven equations make their appearance:

$$
\begin{aligned}
& u_{11} B^{3}+A B^{2} p_{12}=0, \\
& B^{2}\left(2 A^{\prime}+2 u_{11} C-A p_{11}\right)+2 B C\left(u_{22} B-A p_{12}\right) \\
& \quad-A B\left(2 B^{\prime}-B p_{22}-2 C p_{12}\right)=0, \\
& B^{2}\left(u_{11} D-A p_{21}\right)-3 B^{2} D u_{11}+2 B C\left(2 A^{\prime}+2 u_{22} C-A p_{22}\right) \\
& +3 B D\left(u_{22} B-A p_{12}\right)-A B\left(A-D p_{12}+4 C^{\prime}-2 C p_{22}\right)=0, \\
& -3 B D\left(2 A^{\prime}+2 u_{11} C-A p_{11}\right)-2 B C D\left(u_{11}-u_{22}\right) \\
& +3 B D\left(2 A^{\prime}+2 u_{22} C-A p_{22}\right) \\
& \quad+A D\left(2 B^{\prime}-B p_{11}\right)-A B\left(2 D^{\prime}-D p_{22}\right)=0, \\
& -3 B D\left(u_{11} D-A p_{21}\right)-2 C D\left(2 A^{\prime}+2 u_{11} C-A p_{11}\right) \\
& +3 B D^{2} u_{22}-D^{2}\left(u_{22} B-A p_{12}\right)+A D\left(A-B p_{21}+4 C^{\prime}-2 C p_{11}\right)=0, \\
& -2 C D\left(u_{11} D-A p_{21}\right)-D^{2}\left(2 A^{\prime}+2 u_{22} C-A p_{22}\right) \\
& \quad+A D\left(2 D^{\prime}-D p_{11}-2 C p_{21}\right)=0, \\
& -u_{22} D^{3}-A D^{2} p_{21}=0 .
\end{aligned}
$$

Let us assume that both $B$ and $D$ are different from zero. Then the first and last equations give

$u_{11} u_{22} v_{12}+2 u_{11} u_{22}\left(u_{11}-u_{22}\right) p_{12}=0, \quad u_{11} u_{22} v_{21}-2 u_{11} u_{22}\left(u_{11}-u_{22}\right) p_{21}=0$,

or

$$
u_{11} u_{22}\left(u_{11}-u_{22}\right) p_{12}=u_{11} u_{22}\left(u_{11}-u_{22}\right) p_{21}=0 .
$$

But all of the possibilities here suggested give zero values to either $B$ or $D$, or both, except $u_{11}-u_{22}=0$, in which case $S$ is a quadric. But this is only apparently an exception, arising from the fact that in this case the flecnode curve is indeterminant. If two of the straight lines of the second set be taken for the curves $C_{y}$ and $C_{z}$, we have in this case also $B=D=0$.

There remain two possibilities. If $B=D=0$ all of the equations (20) are 
satisfied. If $S^{\prime}$ is not developable, $S$ must then belong to a linear congruence. If $S^{\prime}$ is developable, it is sufficient for $S$ to have one straight line directrix. In either case the derivative cubic degenerates into a straight line, and is therefore obviously an asymptotic curve upon the surface of cubics.

Finally, it might happen that one only of the two quantities $B$ and $D$ is zero. Say $B=0, D \neq 0$. Then the first four equations of $(20)$ are satisfied. The other three become

$$
\begin{gathered}
-4 u_{11} C^{2}+A D p_{12}+A^{2}=0 \\
-2 C u_{11} D-D\left(2 A^{\prime}+2 u_{22} C-A p_{22}\right)+A\left(2 D^{\prime}-D p_{11}\right)=0 \\
-u_{22} D-A p_{21}=0 .
\end{gathered}
$$

From the last of these we find

$$
u_{11} u_{22}\left(u_{11}-u_{22}\right) p_{21}=0,
$$

whence, since $D \neq 0$, follows $u_{22}=0$, and therefore $A=0$. The second equation now gives us $C=0$, which also satisfies the first. We have again $S^{\prime}$ a developable surface, so that the cubic degenerates. Therefore

The derivative cubics of a ruled surface are asymptotic curves, upon the surface formed by their totality, only in the trivial cases when they degenerate into straight lines.

\section{§ 2. Null-system of the derivative cubic.}

A twisted cubic always determines a null-system, i. e., a point-to-plane correspondence with incident elements. Geometrically this correspondence may be set up as follows. An arbitrary plane intersects the curve in three points. The three planes, which osculate the curve in these points, intersect again in a point which is situated in the original plane. This is the point which corresponds to the plane in the null-system of the cubic.

We shall now set up the equations for this null-system. For this purpose it is more convenient to use the equations of the cubic referred to a non-homogeneous parameter $t$.

Let $t_{1}, t_{2}, t_{3}$ be the three values of $t$ which correspond to the three points in which the plane

$$
v_{1} x_{1}+v_{2} x_{2}+v_{3} x_{3}+v_{4} x_{4}=0
$$

intersects the cubic. Then $t_{1}, t_{2}, t_{3}$ are the roots of the cubic equation

$$
B v_{3} t^{3}+\left(A v_{1}+2 C v_{3}+B v_{4}\right) t^{2}+\left(A v_{2}+D v_{3}+2 C v_{4}\right) t+D v_{4}=0 .
$$

Therefore we shall have

$$
\frac{A v_{1}+2 C v_{3}+B v_{4}}{B v_{3}}=-\left(t_{1}+t_{2}+t_{3}\right),
$$




$$
\begin{gathered}
\frac{A v_{2}+D v_{3}+2 C v_{4}}{B v_{3}}=t_{2} t_{3}+t_{3} t_{1}+t_{1} t_{2}, \\
\frac{D v_{4}}{B v_{3}}=-t_{1} t_{2} t_{3} .
\end{gathered}
$$

If we solve these equations for $v_{1} / v_{3}, v_{2} / v_{3}, v_{4} / v_{3}$, then make them homogeneous, and multiply $v_{1} \cdots v_{4}$ by the common factor $A$, we shall find

$$
\begin{array}{ll}
v_{1}=-2 C D+B^{2} t_{1} t_{2} t_{3}-B D\left(t_{1}+t_{2}+t_{3}\right), & v_{3}=A D, \\
v_{2}=-D^{2}+2 B C t_{1} t_{2} t_{3}+B D\left(t_{2} t_{3}+t_{3} t_{1}+t_{1} t_{2}\right), & v_{4}=-A B t_{1} t_{2} t_{3} .
\end{array}
$$

The coördinates of the planes which osculate the curve in the three points $t_{1}, t_{2}, t_{3}$, are, according to $(16)$,

$$
\begin{array}{ll}
u_{1}^{(k)}=B^{2} t_{k}^{3}-3 B D t_{k}-2 C D, & u_{3}^{(k)}=A D, \\
u_{2}^{(k)}=2 B C t_{k}^{3}+3 B D t_{k}^{2}-D^{2}, & u_{4}^{(k)}=-A B t_{k}^{3}
\end{array}
$$

Let $x_{1}, \cdots x_{4}$ be the coördinates of the point of intersection of these three planes. We must then have

$$
\sum_{i=1}^{4} u_{i}^{(k)} x_{i}=0
$$

Solving these equations we find

$$
\begin{aligned}
& x_{1}=A\left(t_{2} t_{3}+t_{3} t_{1}+t_{1} t_{2}\right), \\
& x_{2}=A\left(t_{1}+t_{2}+t_{3}\right), \\
& x_{3}=3 B t_{1} t_{2} t_{3}+2 C\left(t_{2} t_{3}+t_{3} t_{1}+t_{1} t_{2}\right)+D\left(t_{1}+t_{2}+t_{3}\right), \\
& x_{4}=B\left(t_{2} t_{3}+t_{3} t_{1}+t_{1} t_{2}\right)+2 C\left(t_{1}+t_{2}+t_{3}\right)+3 D,
\end{aligned}
$$

and a simple calculation will show that $\sum v_{i} x_{i}=0$, i. e., as we have stated, the point of intersection of the three osculating planes lies in the plane of the three points of osculation.

In our null-system then, the plane (21) and the point (22) correspond to each other. To find the explicit equations for this correspondence, we need only eliminate $t_{1}, t_{2}, t_{3}$ between equations (21) and (22). Denoting by $\omega$ and $\omega^{\prime}$ two proportionality factors, we find:

$$
\begin{aligned}
& \omega v_{1}=\quad+4\left(C^{2}-B D\right) x_{2}+A B x_{3}-2 A C x_{4}, \\
& \omega v_{2}=-4\left(C^{2}-B D\right) x_{1}+*^{*}+2 A C x_{3}-A D x_{4}, \\
& \omega v_{3}=-A B x_{1}-2 A C x_{2}+*+A^{2} x_{4} \\
& \omega v_{4}=2 A C x_{1}+A D x_{2}-A^{2} x_{3}+* ;
\end{aligned}
$$




$$
\begin{aligned}
& \omega^{\prime} x_{1}=*+A^{2} v_{2}+A D v_{3}+2 A C v_{4}, \\
& \omega^{\prime} x_{2}=-A^{2} v_{1}+* v^{*}-2 A C v_{3}-A B v_{4}, \\
& \omega^{\prime} x_{3}=-A D v_{1}+2 A C v_{2}+{ }^{*} \\
& \omega^{\prime} x_{4}=-2 A C v_{1}+A B v_{2}-4\left(C^{2}-B D\right) v_{3}+4\left(C^{2}-B D\right) v_{4},
\end{aligned}
$$

But associated with the null-system we have a linear complex, made up of all of the lines passing through a point, which lie at the same time in the plane corresponding to this point in the null-system. Introduce line coördinates by putting $\omega_{i k}=x_{i} y_{k}-x_{k} y_{i}, \omega_{i k}^{\prime}=v_{i} w_{k}-v_{k} w_{i}$, where $x_{1} \cdots x_{i}$ and $y_{1} \cdots y_{4}$ are coördinates of two points on the line, and where $v_{1} \cdots v_{4}, w_{1} \cdots w_{4}$ are the coördinates of two planes containing the line. Then the equation of the complex may be written in either of the forms

$$
\begin{aligned}
-4\left(C^{2}-B D\right) \omega_{34}^{\prime}-A B \omega_{42}^{\prime}+2 A C \omega_{23}^{\prime} & \\
& -2 A C \omega_{14}^{\prime}-A D \omega_{13}^{\prime}-A^{2} \omega_{12}^{\prime}=0 .
\end{aligned}
$$

This complex becomes special if $A^{2} B D=0$, i. e., only if the cubic degenerates.

To the flecnode $P_{y}\left(x_{2}=x_{3}=x_{4}=0\right)$ corresponds the plane

$$
\left[0,-4\left(C^{2}-B D\right),-A B, 2 A C\right] \text {. }
$$

Therefore, if $C^{2}-B D=0$, i. e., if the derivative cubic is tangent to the generator $g$, the corresponding plane passes through $g$. If $C=0$, i.e., $u_{11} / u_{22}=$ const. the plane passes through $P_{\sigma} P_{y}$. Therefore, if the intersections of the cubic with $g$ and the flecnodes form a harmonic group on $g$, the plane corresponding to each flecnode passes through that point of the derived ruled surface which corresponds to the other.

\section{§3. The osculating linear complex.}

There is another linear complex associated with every generator of a ruled surface, even more important than the one just considered. A linear complex is determined by five of its lines, provided that these have no two straight line intersectors. Let us consider five generators of a ruled surface, $g$ and four others. As the four other generators approach coincidence with $g$, a definite linear complex will in general be obtained as a limit. We shall speak of it as the linear complex osculating $S$ along $g$.

Instead of determining the complex by five consecutive generators of $S$, it will be advisable to determine it by means of two pairs of lines which are reciprocal polars with respect to it. Two such pairs are obviously constituted by 
the flecnode tangent of $g$ and of another generator infinitesimally close to $g$. Let us denote by $g_{x}, f_{x}^{\prime}, f_{x}^{\prime \prime}, g_{x}^{\prime}$ respectively the generator of $S$, the first and the second flecnode tangents and the generator of $S^{\prime}$ which belong to the argument $x$. Similarly we denote by $g_{x+\delta x}$, etc., the corresponding lines belonging to the argument $x+\delta x$, where $\delta x$ is an infinitesimal.

Clearly $f_{x}^{\prime}$ and $f_{x}^{\prime \prime}$ are the directrices of the osculating linear congruence, (determined by four consecutive generators). Therefore all lines intersecting $f_{x}^{\prime}$ and $f_{x}^{\prime \prime}$ belong to the osculating linear complex, which must therefore be of the form

$$
a \omega_{13}+b \omega_{42}=0 \text {. }
$$

Change the parameter $x$ by an infinitesimal quantity $\delta x$. The flecnode tangents $f_{x+\delta x}^{\prime}$ and $f_{x+\delta x}^{\prime \prime}$ must again be the directrices of a linear congruence contained in the complex. We have

$$
y_{x+\delta x}=y_{x}+y_{x}^{\prime} \delta x, \quad \rho_{x+\delta x}=\rho_{x}+\rho_{x}^{\prime} \delta x, \text { etc. }
$$

If we substitute the values of $y^{\prime}, \rho^{\prime}$, etc., from Covariants, equations (21) and (33), we shall find

$$
\begin{aligned}
& 2 y_{x+\delta x}=2 y+\left(\rho-p_{11} y-p_{12} z\right) \delta x, \\
& 2 z_{x+\delta x}=2 z+\left(\sigma-p_{21} y-p_{22} z\right) \delta x, \\
& 2 \rho_{x+\delta x}=2 \rho+\left[u_{11} y+u_{12} z-p_{11} \rho-p_{12} \sigma\right] \delta x, \\
& 2 \sigma_{x+\delta x}=2 \sigma+\left[u_{21} y+u_{22} z-p_{21} \rho-p_{22} \sigma\right] \delta x,
\end{aligned}
$$

where of course $u_{12}$ and $u_{21}$ may be equated to zero, since $C_{y}$ and $C_{z}$ constitute the flecnode curve.

Now clearly, the coefficients of $y, z, \rho, \sigma$ in the expressions

$$
\rho_{x+\delta x}+\lambda y_{x+\delta . x} \quad \text { and } \quad \sigma_{x+\delta x}+\mu z_{x+\delta x}
$$

will be the coördinates of two arbitrary points $P_{1}$ and $P_{2}$, situated on $f_{x+\delta x}^{\prime}$ and $f_{x+\delta x}^{\prime \prime}$ respectively. If (27) is the equation of the osculating complex, the plane which corresponds in it to $P_{1}$ must contain $P_{2}$ for arbitrary values of $\lambda$ and $\mu$. This consideration will enable us to determine the ratio $a: b$.

We find first, remembering that $u_{12}=u_{21}=0$, for $P_{1}$ and $P_{2}$ the coördinates :

\begin{tabular}{c|c|c|c|c} 
& $x_{1}$ & $x_{2}$ & $x_{3}$ & $x_{4}$ \\
\hline$P_{1}$ & $2 \lambda+\left(u_{11}-\lambda p_{11}\right) \delta x$ & $-\lambda p_{12} \delta x$ & $2+\left(-p_{11}+\lambda\right) \delta x$ & $-p_{12} \delta x$ \\
$P_{2}$ & $-\mu p_{21} \delta x$ & $2 \mu+\left(u_{22}-\mu p_{22}\right) \delta x$ & $-p_{21} \delta x$ & $2+\left(-p_{22}+\mu\right) \delta x$
\end{tabular}


But, if we denote by $u_{1}, \ldots, u_{4}$ the coördinates of the plane which corresponds to the point $x_{1}, \cdots, x_{4}$ in the linear complex (27), we find

$$
u_{1}=-a x_{3}, \quad u_{2}=+b x_{4}, \quad u_{3}=a x_{1}, \quad u_{4}=-b x_{2} .
$$

Substituting for $x_{1}, \ldots, x_{4}$ the coördinates of $P_{1}$, and writing down the condition that $P_{2}$ shall lie in the plane corresponding to $P_{1}$, we find that we must have

$$
a(\mu-\lambda) p_{21}+b(\lambda-\mu) p_{12}=0
$$

for arbitrary values of $\lambda$ and $\mu$, i. e., $a: b=p_{12}: p_{21}$.

Therefore, the equation of the osculating linear complex, in the system of coördinates here employed, is

$$
p_{12} \omega_{13}+p_{21} \omega_{42}=0 .
$$

The point-plane correspondence, determined by this complex, is given by the equations

$$
u_{1}=p_{12} x_{3}, \quad u_{2}=-p_{21} x_{4}, \quad u_{3}=-p_{12} x_{1}, \quad u_{4}=p_{21} x_{2} .
$$

Let us consider a point on the generator $g$. There will correspond to it, in this complex, a plane, obviously containing the generator itself. But to every point of $g$ there also corresponds another plane through $g$, viz., the plane tangent to the ruled surface at that point. Clearly, there will exist in general two points on $g$ at which these two planes will coincide. We shall call them the complex points of $g$, and their locus on $S$, the complex curve of the surface. We proceed to determine the complex points of $g$.

The plane corresponding to any point of $g,\left(x_{1}, x_{2}, 0,0\right)$, in the osculating linear complex, has the coördinates

$$
u_{1}=0, \quad u_{2}=0, \quad u_{3}=-p_{12} x_{1}, \quad u_{4}=p_{21} x_{2} .
$$

The coördinates of the plane, tangent to $S$ at the same point, are found most easily by computing the equation of the plane tangent to the osculating hyperboloid $H$ at that point. They are $\left(0,0, x_{2},-x_{1}\right)$. This plane and (30) coincide if and only if $-p_{12} x_{1}: p_{21} x_{2}=x_{2}:-x_{1}$, i. e., if

$$
p_{12} x_{1}^{2}-p_{21} x_{2}^{2}=0 \text {. }
$$

This shows that the complex points and the flecnodes form a harmonic group on every generator of the surface.

If in (7) we put $u_{11}+u_{22}=0$, we find that the derivative cubic intersects $g$ precisely in the complex points. Therefore: if the surface $S^{\prime}$ of the congruence $\Gamma$ is so chosen that it intersects the flecnode surface of $S$ in an asymptotic curve, the surface of derivative cubics will intersect $S$ along its complex curve. 
If $S$ is contained in a linear complex, the complex curve is at the same time an asymptotic curve.

For, we notice in the first place that under our assumptions the factors of the the expression $p_{12} z^{2}-p_{21} y^{2}$ determine the complex points. Let us assume $p_{11}-p_{22}=0$, which we may do without affecting the generality of our argument. Then the condition $\Delta=0$ for a linear complex becomes $p_{12} / p_{21}=$ const. If we now make the transformation

$$
\eta=\sqrt{p_{21}} y+\sqrt{p_{12}} z, \quad \zeta=\sqrt{p_{21}} y-\sqrt{p_{12}} z,
$$

we find that in the transformed system of differential equations, $\pi_{12}=\pi_{21}=0$, the coefficients of this transformed system being denoted by Greek letters. But this proves that $C_{\eta}$ and $C_{\zeta}$ are asymptotic lines on $S$. It is geometrically evident that the tangents of this asymptotic curve will be lines of the linear complex.

LIE seems to have been the first to notice the existence of this special asymptotic curve on a ruled surface belonging to a linear complex. He noticed in 1871 that its determination requires no integration, and that all other asymptotic curves can be obtained by quadratures. ${ }^{*}$ These latter remarks we can also verify at once from our theory. Picard found the same theorems independently in 1877. $t$ These theorems on the determination of all of the asymptotic curves by quadratures if one of them is known, follow at orce from the fact first noted by BonneT that their equation is of the RICCATI form, and had already been explicitly formulated and applied to special surfaces by CLEBSCH. $\ddagger$ It seems that Voss $\S$ was the first to notice that this asymptotic curve and the flecnode curve divide the generators of the surface harmonically. Cremona $\|$ however had already observed this in the special case of a surface with two straight line directrices. The general notion of the complex curve, its relation to the derivative surface and to the surface of derivative cubics do not seem to occur in the literature of the subject.

There always exists a pair of points harmonically conjugate to each of two given pairs. We see easily that the pair

$$
p_{21} y^{2}+p_{12} z^{2}
$$

is thus situated with respect to the flecnodes as well as the complex points. They are therefore the double points of an involution of which the flecnodes

* Lie, Verhandl. d. Ges. d. Wiss. Christiania (1871), Mathematische Annalen, vol. 5 (1872).

† PiCard, These, Paris (1877). See also Darboux, Bulletin (1877), p. 335, and Annales de l'Ecole Normale (1877).

$\ddagger$ Clebsch, Crelle's Journal, vol. 68.

$\S$ Voss, Mathematische Annalen, vol. 8 (1875).

|| Cremona, Anuali di Matematiche (1867-68). 
and the complex points are two pairs. We will call them the involute points, their locus the involute curve. Of course these three pairs cannot be real at the same time.

Consider the expression

$$
\gamma=\theta_{4} E-\theta_{4}^{\prime} C
$$

where

$$
C=u_{12} z^{2}-u_{21} y^{2}+\left(u_{11}-u_{22}\right) y z, \quad E=v_{12} z^{2}-v_{21} y^{2}+\left(v_{11}-v_{22}\right) y z .
$$

It is easy to prove that $\gamma$ is a covariant. Moreover it reduces to (32) under our special assumptions. Therefore, the factors of the covariant $\theta_{4} E-\theta_{4}^{\prime} C$ give the expressions for the involute points in invariant form. If

$$
\theta_{4}^{\prime} u_{12}-\theta_{4} v_{12}=0, \quad \theta_{4}^{\prime} u_{21}-\theta_{4} v_{21}=0,
$$

the combined locus of $C_{y}$ and $C_{z}$ constitutes the involute curve. If however

$$
u_{11}-u_{22}=0, \quad v_{11}-v_{22}=0,
$$

then $C_{y}$ and $C_{z}$ together constitute the complex curve.

We can also write down a covariant whose factors give directly the complex points. It is found by writing down the conditions that a quadratic in $y$ and $z$ shall represent points which divide the pair of the flecnodes, and the pair of involute points harmonically. We find in this way, that the factors of

$$
\begin{aligned}
{\left[\left(u_{11}-u_{22}\right) v_{12}-\left(v_{11}-v_{22}\right)\right.} & \left.u_{12}\right] z^{2}+\left[\left(u_{11}-u_{22}\right) v_{21}\right. \\
& \left.-\left(v_{11}-v_{22}\right) u_{21}\right] y^{2}+2\left[u_{12} v_{21}-u_{21} v_{12}\right] y z
\end{aligned}
$$

represent the complex points. One of the covariants (33) or (36) should be added to the list (Covariants, p. 433), which is incomplete as it stands.

We can easily show that the derivative cubic cannot intersect $g$ in its involute points unless $S$ is a quadric. Moreover if the derivative cubic intersects $g$ in two points which are harmonic conjugates with respect to the complex point, $S$ can only be a quadric. The cubic will however intersect $g$ in two points harmonic conjugates with respect to the involute points, provided that $u_{11}+u_{22}=0$, i. e., provided that $S^{\prime}$ intersects the flecnode surface of $S$ in an asymptotic curve. It then passes through the complex points.

It is further clear, geometrically as well as analytically, that the two complex points, as well as the two involute points, can coincide only if $S$ has a straight line directrix or if the flecnodes coincide. They become indeterminate if $S$ has two straight line directrices.

To every point $P^{\prime}$ of $g^{\prime}$ there corresponds a plane in the osculating linear complex, as well as the plane tangent to $S^{\prime}$ at $P^{\prime}$. When do these planes coincide? 
Let the coördinates of $P^{\prime}$ be $\left(0,0, \alpha_{1}, \alpha_{2}\right)$. The plane, corresponding to $P^{\prime}$ in the complex, has the coördinates $\left(\alpha_{1} p_{12},-\alpha_{2} p_{21}, 0,0\right)$, so that it contains $g^{\prime}$. The coördinates of the plane tangent to $S^{\prime}$ at $P^{\prime}$ are of course the same as those of the plane tangent to $H^{\prime}$ at $P^{\prime}$, which may be obtained from (5), viz: $\left(u_{11} u_{22}^{2} \alpha_{2},-u_{11}^{2} u_{22} \alpha_{1}, 0,0\right)$. These planes coincide if and only if

$$
u_{11} p_{12} \alpha_{1}^{2}=u_{22} p_{21} \alpha_{2}^{2} \text {. }
$$

The corresponding points on $g$ are again harmonic conjugates with respect to the flecnodes, i. e., those asymptotic tangents of $S$ which join $g$ to the points of $g^{\prime}$, the planes corresponding to which in the linear complex are the planes tangent to $S^{\prime}$, are harmonic conjugates with respect to the flecnode tangents.

The planes which correspond to these two points of $g^{\prime}$ in the null-system of the cubic, do not contain $g^{\prime}$.

\$4. Relation of the osculating linear complex to the linear complex of the derivative cubic.

The equations of the two complexes are

$$
\begin{aligned}
& \Omega_{1}=4\left(C^{2}-B D\right) \omega_{12}+A B \omega_{13}+A D \omega_{42} \\
& \\
& \Omega_{2}=p_{12} \omega_{13}+p_{21} \omega_{42}=0 .
\end{aligned}
$$

Their simultaneous invariant is

$$
-2 u_{11} u_{22}\left(u_{11}-u_{22}\right)^{2}\left(u_{11}+u_{22}\right) p_{12} p_{21}
$$

which, leaving aside the cases when $S^{\prime}$ is developable or when $S$ has one or more straight line directrices, vanishes if and only if $u_{11}+u_{22}=0$. Therefore, the osculating linear complex and the complex of the derivative cubic are in involution if the first derivative ruled surface cuts out asymptotic curves on the flecnode surface of $S$, and the cubic passes through the complex points of $g$. Some of our previous theorems are consequences of this.

The two special complexes which are contained in the family

$$
\lambda \Omega_{1}+\mu \Omega_{2}=0,
$$

where $\lambda$ and $\mu$ are constants, are those for which

$$
-3 A^{2} B D \lambda^{2}-2 u_{11} u_{22}\left(u_{11}-u_{22}\right)^{2}\left(u_{11}+u_{22}\right) p_{12} p_{21} \lambda \mu+p_{12} p_{21} \mu^{2}=0,
$$

or, discarding again the case when $S$ has a straight directrix,

$$
-12 u_{11}^{3} u_{22}^{3}\left(u_{11}-u_{22}\right)^{4} \lambda^{2}-2 u_{11} u_{22}\left(u_{11}-u_{22}\right)^{2}\left(u_{11}+u_{22}\right) \lambda \mu+\mu^{2}=0 .
$$

They coincide if

$$
u_{11}^{2} u_{22}^{2}\left(u_{11}-u_{22}\right)^{4}\left(u_{11}+u_{22}\right)^{2}+12 u_{11}^{3} u_{22}^{3}\left(u_{11}-u_{22}\right)^{4}=0,
$$


i. e, if $S^{\prime}$ is developable, if $S$ is a quadric, or if

$$
\left(u_{11}+u_{22}\right)^{2}+12 u_{11} u_{22}=0 \text {. }
$$

We can always choose the independent variable so as to satisfy this condition. In fact, if we change the independent variable by putting $\xi=\xi(x)$, according to Congruence, equation (5), we shall have

$$
\left(\bar{u}_{11}+\bar{u}_{22}\right)^{2}+12 \bar{u}_{11} \bar{u}_{22}=0
$$

if $\xi$ be taken as any solution of the equation

$$
64 \mu^{2}+32\left(u_{11}+u_{22}\right) \mu+\left(u_{11}+u_{22}\right)^{2}+12 u_{11} u_{22}=0,
$$

where

$$
\mu=\{\xi, x\}=\eta^{\prime}-\frac{1}{2} \eta^{2}, \quad \eta=\frac{\xi^{\prime \prime}}{\xi^{\prime}} .
$$

Therefore, there exist two families of $\infty^{1}$ non-developable ruled surfaces in the congruence $\Gamma$ such that the linear congruence, common to the osculating linear complex of $S$ and the linear complex of the derivative cubic, shall have coincident directrices. Any four surfaces of one family intersect all of the asymptotic tangents of $S$ in a point row of constant anharmonic ratio. The two families never coincide unless $\theta_{4}=0$, i. e., unless the flecnode curve intersects every generator in two coincident points. But in this case the congruence is not defined. If $S$ has a straight line directrix this congruence is degenerate.

The coördinates of the plane, which corresponds to a point $\left(x_{1}, x_{2}, 0,0\right)$ of $g$ in the null-system of the cubic, are

$4\left(C^{2}-B D\right) x_{2},-4\left(C^{2}-B D\right) x_{1},-A B x_{1}-2 A C x_{2}, 2 A C x_{1}+A D x_{2}$.

This plane contains $g$ if and only if $C^{2}-B D=0$, i. e., if the derivative cubic is tangent to $g$. It will coincide with the plane tangent to $S$ at this point, if further

$$
-A B x_{1}-2 A C x_{2}=\omega x_{2}, \quad 2 A C x_{1}+A D x_{2}=-\omega x_{1},
$$

where $\omega$ is a proportionality factor, or

$$
A B x_{1}+(2 A C+\omega) x_{2}=0, \quad(2 A C+\omega) x_{1}+A D x_{2}=0,
$$

whence follows $\omega=-A C$ or $-3 A C$. We have therefore

$$
x_{1}: x_{2}=-C: B=-D: C \quad \text { or } \quad x_{1}: x_{2}=C: B=D: C .
$$

These points are harmonic conjugates with respect to the flecnodes.

Therefore, if the derivative cubic is tangent to $g$, there are two points of $g$ whose tangent planes are the planes corresponding to them in the null-system of the cubic. These points and the flecnodes form a harmonic group on $g$. They never coincide with the complex points unless the ruled surface has a straight line directrix. 
The planes, corresponding to a point of $g$ in the null-system of the cubic and in the osculating complex, coincide if

$$
\begin{aligned}
C^{2}-B D & =0, \\
\left(A B-\omega p_{12}\right) x_{1}+2 A C x_{2} & =0, \\
2 A C x_{1}+\left(A D-\omega p_{21}\right) x_{2} & =0,
\end{aligned}
$$

where $\omega$ is a root of the quadratic

$$
\omega^{2}+2 u_{11} u_{22}\left(u_{11}-u_{22}\right)^{2}\left(u_{11}+u_{22}\right) \omega-12 u_{11}^{3} u_{22}^{3}\left(u_{11}-u_{22}\right)^{4}=0,
$$

neglecting again the case when $S$ has a straight line directrix. These two points of $g$ coincide if $\left(u_{11}+u_{22}\right)^{2}+12 u_{11} u_{22}=0$.

More generally, if we write down the conditions that the same plane shall correspond to a point $\left(x_{1}, x_{2}, x_{3}, x_{4}\right)$ in the osculating linear complex and in the complex of the cubic, we shall obtain as the locus of these points two straight lines, the directrices of the congruence common to the two complexes. These conditions are as follows, $x_{1} \cdots x_{4}$ must satisfy the equations :

$$
\begin{array}{r}
*+4\left(C^{2}-B D\right) x_{2}+\left(A B-\omega p_{12}\right) x_{3}-2 A C x_{4}=0, \\
-4\left(C^{2}-B D\right) x_{1}+*+2 A C x_{3}-\left(A D-\omega p_{21}\right) x_{4}=0, \\
-\left(A B-\omega p_{12}\right) x_{1}-2 A C x_{2}+*+A^{2} x_{4}=0, \\
2 A C x_{1}+\left(A D-\omega p_{21}\right) x_{2}-A^{2} x_{3}+*=0,
\end{array}
$$

the vanishing of whose skew-symmetric determinant gives for $\omega$ the quadratic equation (41), which may also be written

$$
\left(A B-\omega p_{12}\right)\left(A D-\omega p_{21}\right)+4 A^{2}\left(C^{2}-B D\right)-4 A^{2} C^{2}=0 .
$$

Let $\omega_{1}$ and $\omega_{2}$ be the two roots of this equation. If we eliminate $x_{3}$ from the first two, $x_{4}$ from the last two equations of (42), if we make use of $(41 a)$ and assume that neither $A$ nor $C^{2}-B D$ is zero, we shall find

whence

$$
\begin{aligned}
-\left(A B-\omega_{k} p_{12}\right) x_{1}-2 A C x_{2}+A^{2} x_{4} & =0, \\
-4\left(C^{2}-B D\right) x_{2}-\left(A B-\omega_{k} p_{12}\right) x_{3}+2 A C x_{4} & =0
\end{aligned} \quad(k=1,2),
$$

$$
\begin{aligned}
-2 C\left(A B-\omega_{k} p_{12}\right) x_{1}-4 A B D x_{2}+A\left(A B-\omega_{k} p_{12}\right) x_{3} & =0 \\
-\left(A B-\omega_{k} p_{12}\right) x_{1}-2 A C x_{2}+A^{2} x_{4} & =0
\end{aligned}
$$

the equations of the two directrices in simpler form than in (42).

A line joining the point $\left(x_{1}, x_{2}, 0,0\right)$ of $g$ to the point $\left(0,0, x_{1}, x_{2}\right)$ of $g^{\prime}$ is a generator of the second kind on $H$. It is not difficult to see that it will intersect the directrix (43) if and only if

$$
-\left(A B-\omega_{k} p_{12}\right)^{2} x_{1}^{2}+4 A^{2} B D x_{2}^{2}=0 .
$$


Hence, the two points, in which either of the directrices of the congruence common to the two complexes intersects the osculating hyperboloid, determine upon this hyperboloid two generators of the second set which are harmonic conjugates with respect to the flecnode tangents.

It also follows easily that the two pairs thus obtained, one corresponding to each directrix, coincide only if $\left(u_{11}+u_{22}\right)^{2}+12 u_{11} u_{22}=0$, i. e., if the directrices themselves coincide. Further if one of these pairs intersects $g$ in the involute points, the same is true of the other pair, so that this can only happen if the directrices coincide. Finally, such a pair of generators of $H$ can pass through the complex points only if $S$ has a straight line directrix, or if $S^{\prime}$ is developable.

The line joining the points $\left(x_{1}, 0, x_{3}, 0\right)$ and $\left(0, x_{1}, 0, x_{3}\right)$ is a generator of the first set on $H$. The coördinates of an arbitrary point of this line are $\left(\lambda x_{1}, \mu x_{1}, \lambda x_{3}, \mu x_{3}\right)$. This line will therefore intersect one of the directrices of the congruence if $x_{1}, x_{3}, \lambda, \mu$ can be determined so as to satisfy the equations

$$
\begin{gathered}
\lambda\left[-2 C\left(A B-\omega_{k} p_{12}\right) x_{1}+A\left(A B-\omega_{k} p_{12}\right) x_{3}\right]-4 \mu A B D x_{1}=0, \\
-\lambda\left(A B-\omega_{k} p_{12}\right) x_{1}+\mu\left(-2 A C x_{1}+A^{2} x_{3}\right)=0,
\end{gathered}
$$

which gives either $A=0, A B-\omega_{k} p_{12}=0$, or

$$
4\left(C^{2}-B D\right) x_{1}^{2}-4 A C x_{1} x_{3}+A^{2} x_{3}^{2}=0 .
$$

The first two cases give either a surface $S$ with a straight line directrix, or else a developable surface $S^{\prime}$. Leaving these cases aside we notice that (45) does not contain $\omega_{k}$ so that if the line on $H$ here considered intersects one of the directrices it intersects the other also. Combining this with our previous result, we see that the following theorem holds.

The four points in which the directrices of the congruence, common to the osculating linear complex and the linear complex of the derivative cubic, intersect the osculating hyperboloid can be grouped into two pairs, such that the line joining the members of each pair shall be a generator of the first set upon the hyperboloid. Upon this generator this pair of points, together with the intersections of the generator with the flecnode tangents, form a harmonic group.

The plane, corresponding to a point $\left(x_{1}, x_{2}, 0,0\right)$ of $g$ in the null-system of the cubic, intersects the flecnode tangents

$$
\begin{aligned}
& f^{\prime} \text { in the point }\left[A B x_{1}+2 A C x_{2}, 0,4\left(C^{2}-B D\right) x_{2}, 0\right], \\
& f^{\prime \prime} \text { in the point }\left[0,2 A C x_{1}+A D x_{2}, 0,4\left(C^{2}-B D\right) x_{1}\right] .
\end{aligned}
$$

The line joining these points is a generator of $H$, if either

$$
A=0, \quad \text { or } \quad C^{2}-B D=0, \quad \text { or } \quad B x_{1}^{2}-D x_{2}^{2}=0 .
$$

Therefore, there exist in general two points on $g$, harmonic conjugates with 
respect to the flecnodes, such that the planes, corresponding to them in the nullsystem of the derivative cubic, pass through a generator of the osculating hyperboloid. If the cubic is tangent to $g$ the null-plane of any point of $g$ contains a generator of $H$, viz, $g$ itself. If $A=0$ likewise, all points of $g$ satisfy the condition of the theorem. Their null-planes all pass through $g^{\prime}$.

§5. Various theorems concerning the flecnode surface. The principal surface of the congruence $\Gamma$.

Let us consider the planes which osculate the flecnode curve of $S$ at $P_{y}$ and $P_{z}$. We have of course $u_{12}=u_{21}=0$. If $x_{1}, x_{2}, x_{3}, x_{4}$ are the coördinates of an arbitrary point of the plane osculating $C_{y}$ at $P_{y}$, we have for the equation of this plane

But

$$
\left|\begin{array}{llll}
x_{1} & x_{2} & x_{3} & x_{4} \\
y_{1} & y_{2} & y_{3} & y_{4} \\
y_{1}^{\prime} & y_{2}^{\prime} & y_{3}^{\prime} & y_{4}^{\prime} \\
y_{1}^{\prime \prime} & y_{2}^{\prime \prime} & y_{3}^{\prime \prime} & y_{4}^{\prime \prime}
\end{array}\right|=0
$$

$$
\begin{aligned}
2 y_{k}^{\prime} & =\rho_{k}-p_{11} y_{k}-p_{12} z_{k}, \quad 2 z_{k}^{\prime}=\sigma_{k}-p_{21} y_{k}-p_{22} z_{k}, \\
-y_{k}^{\prime \prime} & =p_{11} y_{k}^{\prime}+p_{12} z_{k}^{\prime}+q_{11} y_{k}+q_{12} z_{k}, \text { etc. }
\end{aligned}
$$

If we assume again $p_{11}=p_{22}=0$, and substitute into the above equation, it becomes

$$
\left|\begin{array}{ccc}
x_{1}, & x_{2}, & \cdots \\
y_{1}, & y_{2}, & \cdots \\
\rho_{1}-p_{12} z_{1}, & \rho_{2}-p_{12} z_{2}, & \cdots \\
p_{12} \sigma_{1}+2 q_{12} z_{1}, & p_{12} \sigma_{2}+2 q_{12} z_{2}, & \cdots
\end{array}\right|=0 .
$$

But if we introduce again our fundamental tetrahedron $P_{y} P_{z} P_{\rho} P_{\sigma}$, this becomes

$$
p_{12} x_{2}+p_{12}^{2} x_{3}-2 q_{12} x_{4}=0 .
$$

In the same way we find the equation of the plane osculating $C_{z}$ at $P_{z}$ to be

$$
p_{21} x_{1}-2 q_{21} x_{3}+p_{21}^{2} x_{4}=0 \text {. }
$$

To these equations must be added the conditions $u_{12}=u_{21}=0$, if $C_{y}$ and $C_{z}$ are the two portions of the flecnode curve.

Let us assume that this is the case. The osculating planes at $P_{y}$ and $P_{z}$ intersect along a straight line, whose intersections with the osculating hyperboloid may now be found. If $x_{1}, \ldots, x_{4}$ are the coördinates of one of these points of intersection, we find

$$
\begin{aligned}
& p_{12} p_{21}^{2} x_{2}=-2 q_{12} p_{21} x_{1}-\left(p_{12}^{2} p_{21}^{2}-4 q_{12} q_{21}\right) x_{3}, \\
& p_{12} p_{21}^{2} x_{4}=-p_{12} p_{21} x_{1}+2 q_{21} p_{12} x_{3},
\end{aligned}
$$


where the ratio of $x_{1}: x_{3}$ is determined by the quadratic

$$
\left(p_{12}^{2} p_{21}^{2}-4 q_{12} q_{21}\right) x_{3}^{2}+2\left(p_{12} q_{21}+p_{21} q_{12}\right) x_{1} x_{3}-p_{12} p_{21} x_{1}^{2}=0 .
$$

Therefore if $p_{12} q_{21}+p_{21} q_{12}=0$, i. e., if $p_{12} p_{21}=$ const., the two generators of the first kind on $H$ which pass through these points are harmonic conjugates with respect to $g$ and $g^{\prime}$. If

$$
\left(p_{12} q_{21}-p_{21} q_{12}\right)^{2}+p_{12}^{3} p_{21}^{3}=0,
$$

the intersection of the two osculating planes is tangent to the hyperboloid. This latter property is obviously characteristic of a class of ruled surfaces, and can be expressed in invariant form.

If $\theta_{4}^{3} \theta_{9}^{2}+16 \theta_{10}^{3}=0$, the ruled surface $S$ has the following characteristic property. The planes, which osculate the flecnode curve at the two points of its intersection with any generator, intersect in a line which is tangent to the osculating hyperboloid.

To prove this it is sufficient to notice that the invariant equation

$$
\theta_{4}^{3} \theta_{9}^{2}+16 \theta_{10}^{3}=0
$$

reduces to $(49)$ under our special assumptions. Further we notice:

If the independent variable is chosen so that $\theta_{10} / \theta_{4}=$ const., the generator $g^{\prime}$ of the first derived surface is the harmonic conjugate of $g$ with respect to the two generators of the same kind on $H$ which are determined by the points in which the line of intersection of the two osculating planes intersects $H$.

The plane osculating the flecnode curve at $P_{y}$ intersects the flecnode tangent $f^{\prime \prime}$ which passes through $P_{z}$ in the point $\left(0,2 q_{12}, 0, p_{12}\right)$. Similarly, the plane osculating $C_{z}$ at $P_{z}$ intersects $f^{\prime}$ in the point $\left(2 q_{21}, 0, p_{21}, 0\right)$. Therefore, the line joining these points is a generator of $H$ if and only if $p_{12} q_{21}-p_{21} q_{12}=0$, i. e., if $S$ belongs to a linear complex. In other words: the points in which the two planes, osculating the flecnode curve at its points of intersection with any generator, intersect the flecnode tangents are situated upon the same generator of the osculating hyperboloid if and only if the surface belongs to a linear complex.

To each of the two planes $(46 a)$ and $(46 b)$ corresponds a point in that plane by means of the osculating linear complex. These points have the coördinates

$$
\left(-p_{12} p_{21},-2 q_{12}, 0,-p_{12}\right) \quad \text { and } \quad\left(2 q_{21}, p_{12} p_{21}, p_{21}, 0\right) \text {. }
$$

We find that the line joining them intersects $H$ in two points which form a harmonic group with the first two, if $S$ belongs to a linear complex. It is tangent to $H$ if (49) is satisfied. Therefore, if the two planes, osculating the flecnode curve at its two points of intersection with a generator, intersect in a line which is tangent to the osculating hyperboloid, the line joining the two points of these planes which correspond to them in the osculating linear complex is also tangent to the osculating hyperboloid, and conversely. 
We have seen that, under the assumptions $u_{12}=u_{21}=p_{11}=p_{22}=0$, the equations of the sheet $F^{\prime}$ of the flecnode surface assume the form (15). Let us denote by $\mathfrak{u}_{i k}$ the quantities formed for this system according to the same law as are the quantities $u_{i k}$ for the equations of $S$. Then we shall have

$\mathfrak{u}_{12}=0, \quad \mathfrak{u}_{21}=-4\left(q_{11}^{\prime}-q_{22}^{\prime}\right)+8 \frac{\left(q_{11}-q_{22}\right) q_{12}}{p_{12}}, \quad \mathfrak{u}_{11}-\mathfrak{U}_{22}=4\left(q_{11}-q_{22}\right)$.

The curve $C_{y}$ is a branch of the flecnode curve on $F^{\prime}$ as well as on $S$. The other branch is the locus of the point

$$
\mathfrak{U}_{21} y-\left(\mathfrak{U}_{11}-\mathfrak{U}_{22}\right) \rho \text {. }
$$

Now if the transformation $\xi=\xi(x)$ is made, $\rho$ is converted into

$$
\bar{\rho}=\frac{1}{\xi^{\prime}}(\rho+\eta y), \quad \text { where } \quad \eta=\frac{\xi^{\prime \prime}}{\xi^{\prime}} .
$$

Therefore, if a transformation $\xi_{1}=\xi_{1}(x)$ is made such that the derivative surface of $S$ with respect to $\xi_{1}$ shall cut out upon $F^{\prime}$ the second branch of its flecnode curve, $\xi_{1}$ must be so chosen that

$$
\eta_{1}=\frac{q_{11}^{\prime}-q_{22}^{\prime}}{q_{11}-q_{22}}-2 \frac{q_{12}}{p_{12}}
$$

Similarly the sernnd branch of the flecnode curve on $F^{\prime \prime}$ will be obtained by putting

$$
\eta_{2}=\frac{q_{11}^{\prime}-q_{22}^{\prime}}{q_{11}-q_{22}}-2 \frac{q_{21}}{p_{21}} .
$$

The two surfaces of $\Gamma$ thus obtained coincide only if $S$ belongs to a linear complex, i. e., the second branches of the flecnode curves on the two sheets of the flecnode surface of $S$ correspond to each other only if $S$ belongs to a linear complex.

We have seen that the plane osculating $C_{y}$ at $P_{y}$ intersects $f^{\prime \prime}$ in the point $2 q_{12} z+p_{12} \sigma$. The corresponding point on $f^{\prime}$, i. e., the point obtained by finding the intersection of $f^{\prime}$ with the corresponding generator of $H$, is given by $2 q_{12} y+p_{12} \rho$. We find a surface of $\Gamma$ which intersects $f^{\prime}$ and $f^{\prime \prime}$ in these points by making a transformation of the independent variables for which $\eta=2 q_{12} / p_{12}$, or if we denote this special value of $\eta$ by $\eta_{1}$,

If we now denote by $\eta$ the expression

$$
\bar{\eta}_{1}=2 \frac{q_{12}}{p_{12}} \text {. }
$$

we find

$$
\eta=\frac{1}{2} \frac{q_{11}^{\prime}-q_{22}^{\prime}}{q_{11}-q_{22}},
$$

and similarly

$$
\eta=\frac{1}{2}\left(\eta_{1}+\bar{\eta}_{1}\right),
$$

$$
\eta=\frac{1}{2}\left(\eta_{2}+\bar{\eta}_{2}\right) \text {. }
$$


This gives us a very important result. For, we have frequently made use of a normal form for our system of differential equations, in which $\theta_{4}=$ const. But in order to have $\theta_{4}=$ const., we must make precisely the transformation determined by $\eta$. On account of its importance, we will call the surface of $\Gamma$ which is thus obtained, the principal surface of the congruence, and the curves in which it intersects the two sheets of the flecnode surface of $S$ their principal curves. We see then that the principal surface may be constructed as follows.

We consider the flecnodes $P_{y}$ and $P_{z}$ of $g$, the planes $p_{y}$ and $p_{z}$ osculating the flecnode curve at these points, and the points $P^{\prime}$ and $P^{\prime \prime}$ upon the flecnode tangents $f^{\prime}$ and $f^{\prime \prime}$ whose loci are the second branches of the flecnode curves on the two sheets $F^{\prime}$ and $F^{\prime \prime}$ of the flecnode surface. The plane $p_{y}$ intersects $f^{\prime \prime}$ in a certain point to which corresponds a point on $f^{\prime}$ such that the line joining them is a generator of the osculating hyperboloid. This latter point together with $P^{\prime}$ constitute a pair, such that the harmonic conjugate of $P_{y}$ with respect to it is the point in which the principal surface intersects $f^{\prime}$. The intersection with $f^{\prime \prime}$ is found in the same way.

We might also say, that in this way there is determined, upon the generators of $H$, an involution whose double elements are $g$ and the generator of the principal surface.

This construction is especially important for the complete geometrical interpretation of the covariant $C_{3}$. For, the interpretation given in a former paper, (Covariants, p. 450), rests upon the use of the principal surface, since $\theta_{4}$ is assumed to be constant, a fact which appears clearly from the detailed proof of the theorem there given, but which is not mentioned explicitly in its enunciations as it should have been.

By combining a number of our previous results with the notion of the principal surface, we obtain a number of theorems, which may be easily verified. They provide interpretations for the vanishing of certain invariants, and therefore furnish characteristic properties of certain families of ruled surfaces. I write them down without proof.

If $\theta_{15}=0, \theta_{4} \neq 0$, the principal surface is the harmonic conjugate of $S$ with respect to the two ruled surfuces of $\Gamma$ which cut out the second branches of the flecnode curves on $F^{\prime}$ and $F^{\prime \prime}$.

If $\theta_{6} \theta_{4}-9 \theta_{10}=0$, the principal surface intersects $F^{\prime}$ and $F^{\prime \prime}$ along asymptotic curves.

If $4 \theta_{4}^{5}-\left(\theta_{4} \theta_{6}-9 \theta_{10}\right)^{2}=0$, the principal surface is developable.

If $\theta_{15}^{2}-\theta_{9}^{2} \theta_{4}^{3}=0$, the principal surface intersects one of the sheets of the flecnode surface along the second branch of its flecnode curve. It thus intersects both sheets if $\theta_{15}=\theta_{9}=0$.

PARIS, October 6, 1903. 